\title{
2011 Census Overview
}

\section{Glen Watson}

ONS Census Director

Office for National Statistics

\section{Introduction}

There's been a census every ten years in England and Wales since 1801 (except 1941) and I am delighted to be heading up the programme that is in place for the 2011 Census, spanning research and planning, operations and of course, the outputs.

\section{The questionnaire}

We started looking at the questionnaire as far back as 2004, conducting extensive consultation and question testing to make sure that the statistics will be relevant and useful. We were also careful to make sure the questions are acceptable and understandable to the general public.

There were many calls on the limited space in the questionnaire. Although it stretches to 32 pages we are only asking six more questions than in 2001. There are 43 questions for each individual, 14 about the household and 4 about visitors. A number of questions were dropped from 2001.

The census questions were approved by Parliament in the Census (England and Wales) Order in December 2009, following consultation with Welsh ministers. Census regulations were then approved, which set out the duties of the field staff and the provisions to protect confidentiality. The regulations for England came into force in March 2010, and for the first time separate regulations for the census in Wales came into force in June 2010.

\section{Delivery and return}

We decided to extend the 'post-back' process from 2001 to include 'post-out'. So Royal Mail will be delivering the census questionnaires to households. We tried this in the 2007 census test and found that post-out did not reduce response rates very much, but saved a lot of money that we have reinvested into following up non response. 
Also new for 2011 is the opportunity to complete online. This quick, easy and secure facility should appeal to many people, particularly some of our 'hard to count' groups like students and young people in general.

Completion is not the only aspect which has been affected by technology. Our new questionnaire tracking system, together with the national address list created specially for the census, will allow up-to-date management information.

\section{Field staff}

We are using Capita to run our recruitment, pay and training across England and Wales, calling on its considerable experience in local recruitment in order to find the best quality staff for all areas. We need nearly 40,000 staff.

Assisted by technology changes, we have been able to change the field deployment model considerably. Our questionnaire tracking system, which tells us which questionnaires have - and haven't - been returned, will allow us to deploy field staff effectively, concentrating on visiting households which may need extra assistance with returning their questionnaire. Information from the questionnaire tracking system will also enable us to manage variability across a local authority.

\section{Community engagement}

Today's society is very different from that of ten years ago, so we have put in place a wide range of community engagement.

Area managers (157) have been working since August 2010, forging contacts with key groups and organisations in local areas.

We have also employed over 40 community advisors who are working with specific groups, in particular locations, such as Pakistani groups in Bradford and Leeds, and Somali groups in Brent and Camden as well as other areas. Their expertise in engaging with these populations has been disseminated to all census area managers, making sure that best practice in communications can be copied across England and Wales. Community advisors have been using their contacts in, for example, religious groups, business and women's networks, community groups and local organisations, helping spread the census message and explain the benefits of an accurate census.

We have selected and trained our staff carefully, employing skilled contractors to manage the processes, and worked very closely with local authorities and key stakeholder groups the length and breadth of England and Wales. Through these partnerships we have been able to receive wonderfully detailed information to help us form our plans for engagement, and receive assurance that we are doing all that we can to make this census a success.

Further to this, our advertising campaign includes TV, outdoor advertising and digital media. The creative platform for the campaign is centred around the 'Help tomorrow take shape' concept. Purple origami items (building on the census's purple $\mathrm{C}$ logo identity) are crafted into objects such as buses and hospitals, representing the public services that are prioritised and planned using census statistics. 


\section{Accessibility}

This year, the census will be supported by over 50 dedicated language lines and these will be widely promoted, including in the leaflet which comes with each questionnaire. Further to this, we have worked with national organisations to create a suite of accessibility materials to aid people who wish to complete their census questionnaire independently. These materials include online help, British Sign Language and audio clips on the website, large print questionnaires and an easy read leaflet.

\section{Processing}

We're estimating that 600 million pages of census questionnaires will be scanned with the data being captured and coded in our 180,000 square foot secure warehousing and processing facility. This is in Trafford Park, Manchester, where our contractors have employed some 1,300 people to be operational during March to December 2011. The census helpline (contact centre) is also based in Manchester where around 400 people are employed to answer calls from the general public should they require clarification about completing their questionnaire.

\section{Census Coverage Survey}

The Census Coverage Survey (CCS) takes place from May to June and we will be visiting about 300,000 addresses, conducting brief doorstep interviews to re-enumerate a sample of postcodes. The CCS is designed to find the sorts of households and persons that the census may have missed. We can then identify who was counted in just the census, just the CCS, or in both the census and the CCS. We can use this information to estimate the population missed by the census in the CCS areas, and then extrapolate to the whole country to estimate the total population by local authority and key characteristics. The census results are then adjusted to reflect these estimates and the characteristics of those missed, by imputing missed households and missed persons.

\section{Quality assurance}

While all of the operational elements have been pulled together over the last 12 months, we have also been putting a significant quality assurance process into place and building the systems to ensure that, when the data arrives from the processing plant, we are ready to quality assure it.

QA studies were carried out to identify the data sources that were held by local authorities that we could use for this process. The studies took place during 2010 and this process has been extremely valuable in understanding the value of datasets which local authorities have provided.

Census methodology tutorials (providing a clear explanation of how census estimates are produced and quality assured) were run with local authorities (during late 2010) to ensure that users of census data have confidence in the approach taken by ONS.

Further to this, an independent review has taken place of the entire coverage adjustment and quality assurance process, with the result published in February 2011. 


\section{Outputs}

New technical solutions are being harnessed for 2011 Census outputs, the first set of which will be released around mid-2012. Data visualisation, for example maps and animated charts, will be used more extensively and a new ONS website is being launched with improved search facilities.

ONS does not expect to publish 2011 Census printed reports and will move instead to a contemporary internet model. Our web strategy proposes to integrate the functionality of the NeSS, NOMIS, and ONS web sites onto a single site in the long term.

The 2011 Census outputs project recently launched a trial Application Programming Interface (API) that is available to selected external websites. Three partners are now trialling the accessing of data through the API and developing web applications, with a public facing API planned for this summer. Throughout 2011 work will continue to develop the API into a full service that will ultimately provide data to a high number of web applications across the internet.

We have consulted widely with census users during this process, to make sure that our outputs reflect user requests and meet expectations. We have taken great care to talk to a wide range of users including members of the public, government departments, unitary, county and district councils, academics, businesses and other large census user organisations.

Our output work very much reflects advances in technology and responds to the challenges presented by users after 2001 , to take the dissemination of statistics to a new level. This includes a consolidated UK 'one-stop-shop' for UK level census statistics.

\section{Challenges}

Of course, it has not been easy. We have faced some rather gruelling hurdles along the way - as any project spanning ten years would do; but I believe that we are well prepared for the challenges ahead. While we tested our processes and ways of working by rehearsing in 2009 (in the three challenging areas of Isle of Anglesey, Lancaster and Newham), the true success of 2011 will be measured by the response rates, the effectiveness of our coverage adjustment and quality assurance, and the quality of the final outputs. The biggest danger to a project such as this is public apathy, but with all the measures, care and time we have taken to engage with groups across England and Wales, I am confident that we will achieve our response targets and that, like all previous censuses, the 2011 statistics will go on to play their part in key decisions for years to come. 